\title{
CONTENT PRESERVING WATERMARKING FOR MEDICAL IMAGES USING SHEARLET TRANSFORM AND SVD
}

\author{
M. N. Favorskaya ${ }^{a} *$, E. I. Savchina ${ }^{a}$ \\ ${ }^{\text {a }}$ Institute of Informatics and Telecommunications, Reshetnev Siberian State Aerospace University, 31, Krasnoyarsky Rabochy av., \\ Krasnoyarsk, 660037 Russian Federation - favorskaya@sibsau.ru, savchina.ei@gmail.com
}

Commission II, WG II/10

KEY WORDS: Watermarking, Medical images, Shearlet transform, SVD

\begin{abstract}
:
Medical Image Watermarking (MIW) is a special field of a watermarking due to the requirements of the Digital Imaging and COmmunications in Medicine (DICOM) standard since 1993. All 20 parts of the DICOM standard are revised periodically. The main idea of the MIW is to embed various types of information including the doctor's digital signature, fragile watermark, electronic patient record, and main watermark in a view of region of interest for the doctor into the host medical image. These four types of information are represented in different forms; some of them are encrypted according to the DICOM requirements. However, all types of information ought to be resulted into the generalized binary stream for embedding. The generalized binary stream may have a huge volume. Therefore, not all watermarking methods can be applied successfully. Recently, the digital shearlet transform had been introduced as a rigorous mathematical framework for the geometric representation of multi-dimensional data. Some modifications of the shearlet transform, particularly the non-subsampled shearlet transform, can be associated to a multi-resolution analysis that provides a fully shift-invariant, multi-scale, and multi-directional expansion. During experiments, a quality of the extracted watermarks under the JPEG compression and typical internet attacks was estimated using several metrics, including the peak signal to noise ratio, structural similarity index measure, and bit error rate.
\end{abstract}

\section{INTRODUCTION}

The rapid development of medical information systems is highly crucial due to its significance in clinical diagnosis, treatment, research, and other applications. Two main concepts, such as the Hospital Information System (HIS) and the Picture Archiving and Communication Systems (PACS), are based on the DICOM standard (DICOM, 2016), starting from 1993. The DICOM standard provides a file format for storage and transmission of digital medical images in health care centers. The DICOM information model includes four identification entities called as "Patient" (with attributes Patient ID, Patient Name, Patient Birth Day, Patient sex), "Study" (with attributes Study ID, Study Date, Study Time, Referring Physician, Accession Number), "Series" (with attributes Series Number, Modality Type, Manufacturer, Institution Name), and "Image" (with attributes Image Number, Image Type). The generalized DICOM concept provides the outstanding possibilities in teleconsulting, tele-diagnosis, tele-surgery, etc., as well as in support of Electronic Health Record (EHR) technology that has replaced the inefficient paper records paradigm. However, these advances provoke to increase the new risks for an illegal use of medical information, when someone can easily manipulate with the digital medical images.

Original motivation of the watermarking technique was to protect a copyright but it can be applied in many applications, including a medicine. The MIW is a particular case with high requirements to the security, confidentiality, and integrity. The MIW is applied to hide the confidential personal information, authenticate the images by tracing of the origin of an image and/or investigate the integrity by detection of possible changes in a medical image.

The main requirement for the watermarked medical images is still to conform to the DICOM format (Acharya et al., 2004), in particular such images ought to be suitable for the following compression with losses according to the JPEG compression standard. Also it is required to use the eight bits of ASCII code in the encrypted text file.

All watermarking techniques can be divided in the spatial and frequency domains, among which the most popular ones are the following:

1. Spatial domain: a secure EHR data-hiding technique based on the bipolar multiple-base conversion; the Least Significant Bit (LSB) plane replacement scheme for interleaving patient information (textual and graphical) in the medical images; a reversible authentication of the DICOM images based on the Region of Interest (ROI)

2. Frequency domain: a replacement of the last bits of the Discrete Cosine Transform (DCT) coefficients from the middle frequency range; a robust watermarking method for embedding the encrypted patient information and digital signature of the medical practitioner into the border pixels of images using the Discrete Wavelet Transform (DWT)

Recently, the shearlet transform had been introduced as a rigorous mathematical framework for the geometric representation of multi-dimensional data (Easley et al., 2008). Note that the shearlet transform is actively applied for the

\footnotetext{
* Corresponding author
} 
medical images improvement. The shearlet transform can be associated to a multi-resolution analysis that provides a fully shift-invariant, multi-scale, and multi-directional expansion. In current research, the Digital Shearlet Transform (DST) was used as a promising embedding technique for the MIW.

The remainder of this paper discusses the following components. In Section 2, the related work is represented. Section 3 investigates a pre-processing of medical dental images. The proposed watermarking technique is introduced in Section 4. The experimental results are discussed in Section 5. Section 6 contains the conclusions.

\section{RELATED WORK}

The requirements to the MIW are conventional and include the imperceptibility to a human eye, robustness, the Electronic Patient Record (EPR) payload that can be hidden in the image, reversibility, and less complex embedding/extraction schemas for high computational speed. However, two special requirements deal with the authenticity, when the data content ought to be accessible to the legal users only (for this purpose, a cryptography is used), and intactness of the ROI because the damaged ROIs have serious consequences with respect to a patient's health. However, many algorithms utilize the both ROI and Region Of Non-Interest (RONI) for embedding (Manasrah and Al-Haj, 2008).

The watermarking methods for the medical images are categorized into the robust, fragile, and semi-fragile approaches. The robust methods were used primarily for the copyright protection and content tracking. This is the most promising approach that embeds a watermark with the copyright and patient information around the ROI in an image (Wakatani, 2002). The fragile methods are used to determine whether an image has been modified. Usually, a fragile watermark has a view of checksums, pseudo-random sequences in the LSB plane, or hash functions and is destroyed if the image was manipulated. Also the fragile invertible authentication methods were proposed for medical images (Fridrich et al., 2001). The semi-fragile methods combine the advantages of both robust and fragile approaches, i.e., the robustness to some changes and a possibility to localize the altered regions.

Although the main idea to embed the watermarking information around the ROIs did not changes since 2000s, the watermarking techniques had been developed rapidly from the Gabor transform (Kong and Feng, 2001), the DCT (Osborne et al., 2004; Puech and Rodrigues, 2004), and the DWT (Giakoumaki et al., 2003) to the enhanced hybrid watermarking methods (Blackledge and Al-Rawi, 2014; Mohananthini and Yamuna, 2015).

Some algorithms separate the embedded data into several watermarks, such as the robust watermark for doctor's digital signature, caption watermark for patient's personal and diagnostic information, index watermark for image retrieval, and fragile reference watermark for integrity control (Jianfeng et al., 2015). Sometimes, an index watermark is excluded from such multiple watermark schemas. Badran et al. (Badran et al., 2009) suggested a multiple watermarks embedding algorithm that embedded four types of watermarks: a robust watermark in the RONI in the wavelet domain, a fragile watermark in the ROI in the spatial domain, the physician's digital signature, and the EPR in the RONI in wavelet domain depending on a private key. The authors claimed that their algorithm, which was used for MRI brain images with a brain tumor as the ROI, is robust to the JPEG compression, the ROI removal, geometrical attacks, and some types of noise (Gaussian, Poisson, salt and pepper, and speckle). The robust watermark was an image of a brain tumor on the case that someone removes or adds additional tumor to the original image. The physician's digital signature and the fragile watermark are the binary Pseudo-Noise (PN) sequences with various length generated by a different private seed and generator. During extraction, these sequences are compared with many PN sequences generated from the same generator and the closest ones are considered the extracted sequences. Then the extracted sequences are analyzed on the influence of the possible attacks. The EPR is formed by the binary representation of ASCII codes.

The EPR includes the patient record details like surname, name, sex, age, address, results of examination/diagnosis, time taken, etc. It also contains the medical diagnosis report of the patient, digital signatures of the physician, some specific patient's ID, and many other details (Nambakhsh et al., 2011). Often the EPR and images (with volume achieved $15 \mathrm{MB}$ each one) are stored separately that leads to larger volume storage and a necessity of data transferring. Therefore, a compression of the medical images with minimal file size in order to minimize a transmission time through wireless communications has a significant meaning. The concept of the ROI watermarking in highlights of the JPEG compression was proposed by Wakatani (Wakatani, 2002), who placed the signature information into the Region Of Background (ROB). The details of the JPEG compression and verification after transmission of the ROI and the ROB are well discussed in research of Osborne et al. (Osborne et al., 2004).

\section{PRE-PROCESSING OF DENTAL IMAGES}

Before a watermarking, the medical images ought to be segmented in the ROI and the RONI. The ROI is an area, which represents the main area of interest for the medical practitioner and ought to be stored without any distortions. The RONI is a place for embedding of the textual or graphic information as a binary bit sequence, usually containing $\{1,-1\}$ values. Often a selection of the ROI/RONI is implemented manually by a doctor. Sometimes such selection is executed automatically using geometric primitives like rectangle, ellipse, and polygon (Guo and Zhuang, 2009; Das and Kundu, 2012). It is evident that often the ROI location is close to the center of an image. Therefore, the border areas as the RONI are the container for data embedding. Nyeem et al. (Nyeem et al., 2015) formulated the RONI selection criteria in a following manner:

1. The RONI should have no impact on the medical objectives, for which the image was obtained

2. The RONI should provide the required capacity to accommodate the payload

3. The RONI should keep the distortion at the minimum level

4. The RONI should have the minimum computational cost

The mentioned above criteria are the conventional ones but the RONI selection depends strongly from a type of medical images. For example, the computed tomography scans of brain depicted in Figure 1a provide the explicit RONI, while the computed radiography scans of teethes (Figure 1b) demonstrate the implicit RONI. 

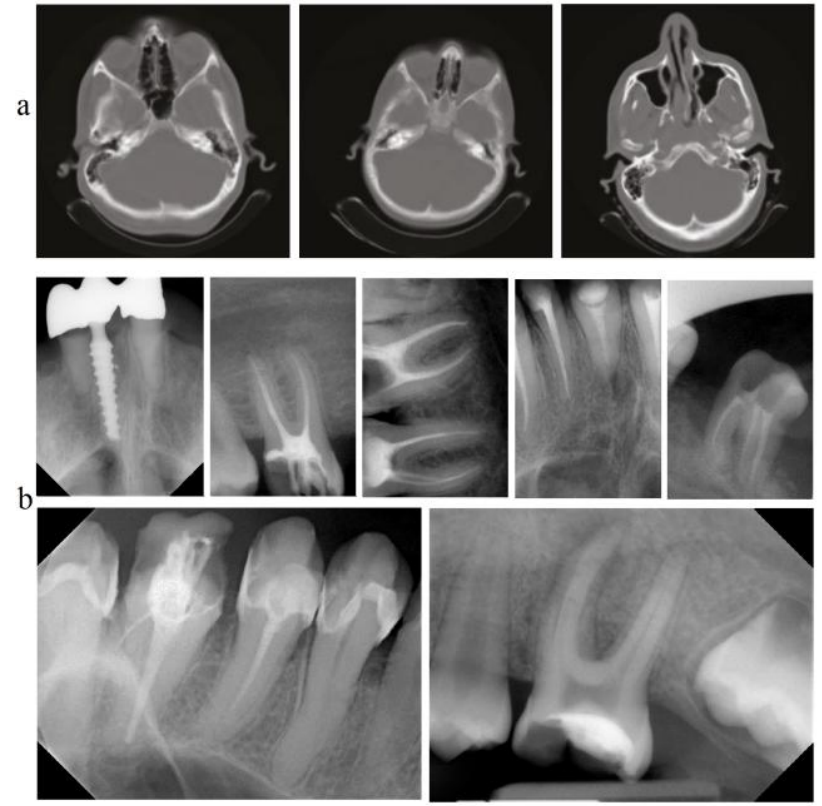

Figure 1. Examples of medical images: a the computed tomography scans of brain, $b$ the computed radiography scans of teethes

The additional preference of the DST application is a possibility to improve a visibility of all contours in a whole medical image after a watermark extraction (Yi et al., 2009). The shearlet coefficients are closely related to the geometric features. This means that such features can be controlled by the magnitude values of shearlet coefficients. Moreover, the adaptive nonlinear mapping based on the shearlet transform permits to amplify the weakly visible edges (that have a great meaning for the disease diagnostics), keeping the perceptible edges intact. Easley and Labate (Easley and Labate, 2012) pointed on the transform thresholding algorithms, including hard thresholding algorithm that sets to zero the coefficients, whose absolute values fall below a certain threshold $T$ (the value of $T$ depends on the standard deviation of the noise $\sigma$ ), and soft thresholding algorithm, where the coefficients are modified by the shrinkage function: $\operatorname{shr}(c)=\operatorname{sgn}(c) \max (|c|-T, 0)$. The hard thresholding is an "all or nothing procedure", when values above the threshold are kept and values below the threshold are deleted. The soft thresholding produces the smooth transitions between the original and the deleted values, when values slightly below the threshold are not removed but attenuated. The main challenge is to find an appropriate value of $T$. Note that sometimes the contrast limited adaptive histogram equalization is required in order to increase the local contrast of the ROIs (Pizer et al., 1987).

A preparing of the watermark information includes a separate processing of the doctor's digital signature, the EPR, watermark with the ROI, and fragile watermark. The doctor's digital signature and fragile watermark are two random binary sequences with different length. The EPR may be extracted from the DICOM file or prepared manually with following coding, e.g., by the Race Integrity Primitives Evaluation Message Digest RIPEMD-160 (Dobbertin et al., 1996).

\section{WATERMARKING TECHNIQUE}

In this section, the short theory of the DST and Singular Value Decomposition (SVD) is discussed in Section 4.1, while the proposed embedding and extraction algorithms based on the DST and the SVD are described in Sections 4.2-4.3, respectively.

\subsection{Shorty Theory of the DST and SVD}

The use of the DST provides a variety of sub-bands for inserting the secret data due to the shearlets' multi-resolution property. These sub-bands are correlated in the DWT but not correlated in the DST that makes a watermarking more secure procedure (Kumar, 2015). More, the modification of DST called as the non-subsampled shearlet transform is a fully shift-invariant, multi-scale, and multi-directional expansion of the shearlet transform (Wang et al., 2016).

Consider the concept of the DST shortly. For an image $I$, the shearlet transform is a mapping in a view of equation 1 :

$$
I \rightarrow S H_{\psi} I(a, s, x)
$$

where $\quad a=$ a scale, $a>0$

$$
s=\text { an orientation, } s>Z
$$

$x=$ a location

The shearlets are created by dilation, shear, and translation. In discrete form for each mother function $\psi=L^{2}\left(R^{2}\right)$, it has provided by equation 2 :

$$
\psi_{j, k, l}=|\operatorname{det} \mathbf{A}|^{\frac{j}{2}} \psi\left(\mathbf{B}^{k} \mathbf{A}^{j} x-m\right): j, k \in Z, \quad l \in Z^{2},
$$

where

$$
\mathbf{A}=\left(\begin{array}{ll}
a & 0 \\
0 & \sqrt{a}
\end{array}\right) \quad \mathbf{B}=\left(\begin{array}{ll}
1 & s \\
0 & 1
\end{array}\right)
$$

The matrices $\mathbf{A}$ and $\mathbf{B}$ are the reversible $2 \times 2$ matrices, which represent the dilation and geometrical transforms like rotation and shear. Notice that the continuous shearlet transform can be discretized by its main parameters, such as the scale, shear, and translation parameters. The mathematical properties of shearlets were summarized by Easley et al. (Easley et al., 2008) as mentioned below:

1. The shearlets are well localized

2. The shearlets satisfy the parabolic scaling

3. The shearlets demonstrate highly directional sensitivity

4. The shearlets are spatially localized

5. The shearlets are optimally sparse

The SVD is often employed in digital watermarking due to the following reasons (Makbol and Khoo, 2014):

1. The singular values involve the maximum energy properties of an image

2. The variations of singular values of an image are small, when an image is under a little distortion

3. The singular values depict the natural algebraic image properties 
The SVD of an image $I$ with sizes $L \times M$ under 2-level DST is given by $\mathbf{A}=\mathbf{U S V}^{\mathrm{T}}$, where $\mathbf{U}$ and $\mathbf{V}$ are unitary (orthogonal) matrices, and $\mathbf{S}=\operatorname{diag}\left(\lambda_{i, i}\right)$ is a diagonal matrix of singular values $\lambda_{i, i}$ that are sorted in decreasing order.

$$
\begin{aligned}
& \lambda_{1,1} \geq \lambda_{2,2} \geq \ldots \geq \lambda_{r, r}>\lambda_{r+1, r+1}= \\
& \lambda_{r+2, r+2}=\ldots=\lambda_{l, m}=0,
\end{aligned}
$$

where $\quad r=$ a rank of the matrix $\mathbf{S}$ matrix $\mathbf{S}$

$l, m=$ the indexes correspond to the sizes of the

The last bits of $\lambda_{i, i}$ singular values contain the embedded binary bits.

\subsection{Embedding Algorithm}

The principal embedding algorithm is summarized as follows:

1. If it is necessary, process the original medical image improving the edges, luminance, and contrast

2. Detect the ROI/RONI. Create a mask of embedding strength on a grid with $4 \times 4$ or $8 \times 8$ non-overlapping blocks in dependence of resolution of the original medical image. This information is a part of a secret key

3. Create the doctor's digital signature and fragile watermark using random seed and generator. These parameters are the part of a secret key

4. Specify and encrypt the confidential attributes in the EPR

5. Specify the ROI as a main watermark

6. Convert the doctor's digital signature, fragile watermark, the EPR, and main watermark into the generalized binary stream

7. Apply the 2-level DST to the selected nonoverlapping blocks in the original medical image

8. Loop (processing of a current block): apply the SVD to the coefficients of the DST in a current block and replace the last significant bits in a sequence of eigenvalues $\lambda_{1,1}, \lambda_{2,2}, \ldots, \lambda_{l, m}$ by a binary sub-sequence of a generalized binary stream forming the scrambled image

9. Apply the inverse DST to the watermarked image

The output information is the watermarked image and a secret key.

\subsection{Extraction Algorithm}

The principal extraction algorithm includes the follow steps:

1. Select the corresponding regions in the watermarked image using a secret key.

2. Apply the 2-level DST to the selected $4 \times 4$ or $8 \times 8$ non-overlapping blocks in the original medical image

3. Loop (processing of a current patch): apply the SVD to the coefficients of the DST in a current block, extract the last significant bits in a sequence of eigenvalues $\lambda_{1,1}, \lambda_{2,2}, \ldots, \lambda_{l, m}$, and put these last significant bits into the generalized binary stream

4. Apply the inverse DST to the extracted host image.

5. Split up the generalized binary stream into the doctor's digital signature, fragile watermark, the EPR, and main watermark
6. Apply the corresponding transforms using a secret key in order to obtain a confirmation of the doctor's digital signature, presence/absence of the attacks (using a fragile watermark), the extracted textual EPR, and the restored image of the ROI

7. Apply a post-processing of the restored image if it is necessary

The output information is the extracted textual EPR and the restored image with the non-distorted ROI.

\section{EXPERIMENTAL RESULTS}

For experiments, the computed radiography dental images were chosen. A volume of embedded information in a single dental image was estimated. Also some results of a host image and watermark quality under the typical transmission attacks, such as the unapproved rotation, translation, and scaling, were obtained.

Different similarity measures, such as Signal to Noise Ratio (SNR), Peak SNR (PSNR), Weighted PSNR (WPSNR), Mean Square Error (MSE), Structural Similarity Index Measure (SSIM), Mean SSIM (MSSIM), Bit Error Rate (BER), Correlation Quality (CQ); Normalized Cross Correlation (NCC), are recommended to estimate a quality of the watermarked images (Nyeem et al., 2013). Notice that in MIW applications, a perceptual similarity ought to be very high to avoid any risk of misdiagnosis. The PSNR metric is used frequently as an objective image quality metric but it does not consider characteristics of the Human Visual System (HVS). The SSIM metric is a region-based metric considering the HVS properties. This metric is promising for testing the medical images because it focuses on the local image similarities. Generally, the SSIM compares the similarity in the luminance, contrast, and structure of the corresponding image regions in a view of equation 5 :

$$
\operatorname{SSIM}\left(r, r^{\prime}\right)=\operatorname{LM}\left(r, r^{\prime}\right)^{\alpha} \cdot C N\left(r, r^{\prime}\right)^{\beta} \cdot \operatorname{ST}\left(r, r^{\prime}\right)^{\gamma},
$$

where $\quad r, r^{\prime}=$ pair of corresponding regions in two images $I$ and $I^{\prime}$

$$
\begin{aligned}
& L M=\text { a luminance } \\
& C N=\text { a contrast } \\
& S T=\text { a structure } \\
& \alpha, \beta, \gamma=\text { the weights for the luminance, contrast, and }
\end{aligned}
$$
structure components, respectively, $\alpha \geq 1, \beta \geq 1, \gamma \geq 1$

Usually equation 5 is simplified subject to $\alpha=\beta=\gamma=1$ :

$$
\begin{aligned}
& \operatorname{SSIM}\left(r, r^{\prime}\right)= \\
& \left(\frac{2 \mu_{r} \mu_{r^{\prime}}+C_{1}}{\mu_{r}^{2}+\mu_{r^{\prime}}^{2}+C_{1}}\right) \cdot\left(\frac{2 \sigma_{r} \sigma_{r^{\prime}}+C_{2}}{\sigma_{r}^{2}+\sigma_{r^{\prime}}^{2}+C_{2}}\right) \cdot\left(\frac{\sigma_{r r^{\prime}}+C_{3}}{\sigma_{r} \sigma_{r^{\prime}}+C_{3}}\right),
\end{aligned}
$$

where $\quad \mu_{r}, \mu_{r^{\prime}}=$ the local means of $r$ and $r^{\prime}$

$\sigma_{r}$ and $\sigma_{r^{\prime}}=$ the local standard deviations of $r$ and $r^{\prime}$

$\sigma_{r r^{\prime}}=$ the covariance of $r$ and $r^{\prime}$

$C_{1}, C_{2}$, and $C_{3}=$ the small positive constants to avoid a numerical instability given by equation 7 :

$$
C_{1}=\left(K_{1} L\right)^{2}, \quad C_{2}=\left(K_{2} L\right)^{2}, \quad C_{3}=C_{2} / 2,
$$

where $L=$ the dynamic range of the pixel values, $L=255$ for 8 bits/pixel grayscale images 

and $K_{2}=0.03$

$K_{1}$ and $K_{2}=$ two scalar constants, usually $K_{1}=0.01$

The resulting SSIM is given by equation 8 :

$$
\operatorname{SSIM}\left(r, r^{\prime}\right)=\left(\frac{2 \mu_{r} \mu_{r^{\prime}}+C_{1}}{\mu_{r}^{2}+\mu_{r^{\prime}}^{2}+C_{1}}\right) \cdot\left(\frac{\sigma_{r r^{\prime}}+C_{2}}{\sigma_{r} \sigma_{r^{\prime}}+C_{2}}\right)
$$

Equation 8 satisfies the following conditions: symmetry $\operatorname{SSIM}\left(r, r^{\prime}\right)=\operatorname{SSIM}\left(r^{\prime}, r\right)$, boundedness $\operatorname{SSIM}\left(r, r^{\prime}\right) \leq 1$, and unique maximum $\operatorname{SSIM}\left(r, r^{\prime}\right)=1$ if and only if $r=r^{\prime}$. The final SSIM (MSSIM) is obtained as the average of the local SSIMs. The BER metric evaluates the similarity between the original EPR data and the recovered EPR data, while the NCC metric calculates the likeness of the extracted and original watermarks in a signature watermarking.

During experiments, different types of watermarks in the host image were embedded. Thus, the examples of the EPRs are depicted in Figure 2, while the test medical images with specified ROIs called as ROI1 and ROI2 are presented in Figure 3. These ROIs were segmented manually by the doctor and outlined by yellow rectangles.

$\begin{array}{lll}\begin{array}{ll}0015234 \\ \text { J.K.Johnson }\end{array} & \text { 0014513 } \\ \text { J.Smith } \\ \text { male 42 } & \text { b } \begin{array}{l}\text { male } 27 \\ \text { 12 White street }\end{array} & 3 \text { Black street } \\ \text { Dia: periodontitis } & \text { Dia: caries } \\ 24.02 .17 & 27.02 .17\end{array}$

Figure 2. Examples of the EPR watermarks: a EPR1, b EPR2
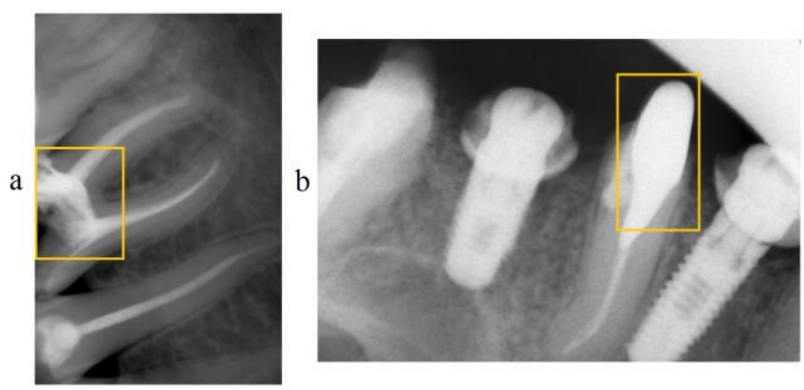

Figure 3. Test dental images with the specified ROIs outlined by yellow rectangles: a host image with ROI1, b host image with ROI2

Robustness of the watermarks was tested under various attacks, possible during the internet transmission. First, the watermarks were embedded in a host image. Second, the attacks with various parameters were modeled. Third, the extracted watermarks were compared with the original watermarks using some metrics, which estimated a quality of the extracted watermarks. A comparison of textual watermarks (EPR1 and EPR2) was realized using the BER metric (due to their binary representation), while a comparison of the ROIs (ROI1 and ROI2) was implemented using three metrics, such as the PSNR, SSIM, and BER.

The estimations of extracted watermarks under rotation distortions subject to $\pm 5^{\circ}, \pm 10^{\circ}, \pm 15^{\circ}$, and $\pm 20^{\circ}$ rotations are placed in Table 1. The watermarked images under rotation attacks, as well as the extracted textual EPR and ROI watermarks are depicted in Figure 4. The obtained quality values demonstrate a high robustness of the designed algorithm to rotations lesser $\pm 15^{\circ}$. If a value of rotating angle becomes more, then a number of error bits in a watermark increases intensively.

\begin{tabular}{|l|c|c|c|c|}
\hline \multicolumn{1}{|c|}{ Metric } & \multicolumn{4}{|c|}{ Rotation, $^{\circ}$} \\
& \pm 5 & $\pm 10 \quad \pm 15$ & \pm 20 \\
BER, \% & 0.8 & 1.8 & 2.6 & 3.7 \\
& \multicolumn{5}{|c|}{ EPR1 } \\
SSIM, \% & 99.3 & 97.7 & 91.4 & 87.0 \\
PSNR, dB & 38.5 & 36.4 & 34.8 & 29.5 \\
BER, \% & 1.1 & 1.8 & 1.9 & 3.8 \\
& \multicolumn{4}{|c|}{ EPR2 } \\
BER, \% & 1.3 & $2.0 \quad 2.1$ & 4.6 \\
& \multicolumn{4}{|c|}{ ROI2 } \\
SSIM, \% & 99.4 & 97.3 & 93.3 & 86.1 \\
PSNR, dB & 39.0 & 36.4 & 35.1 & 30.6 \\
BER, \% & 1.4 & 1.7 & 1.9 & 5.2 \\
\hline
\end{tabular}

Table 1. Estimations of the extracted EPR and ROI watermarks under rotation distortions

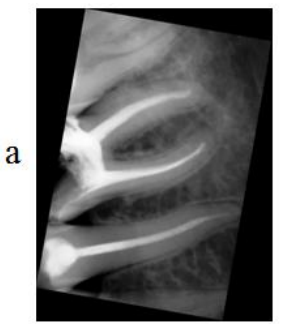

$001 \mu 2 \mathrm{i} 4$

J.K.JnhnsHf

male 42

$12 \mathrm{~W}^{\circ}$ ite strget

Dia: pgriodonpiti3

24.02.17
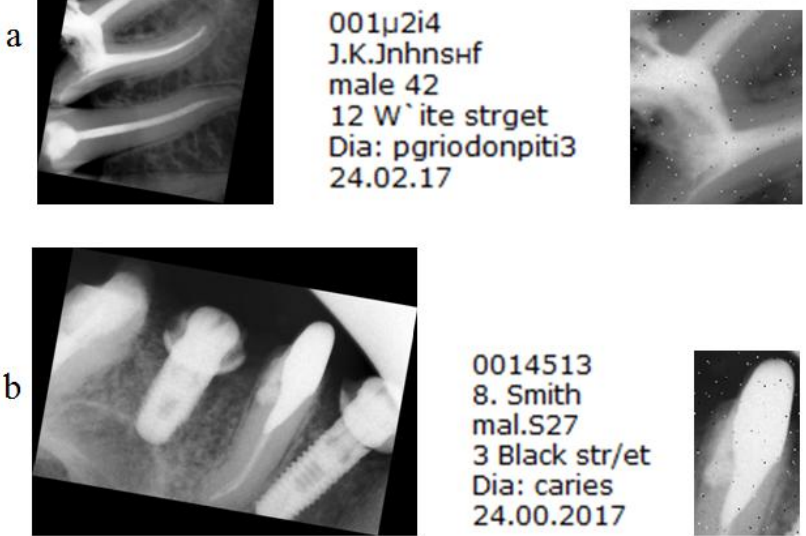

0014513

8. Smith

mal.S27

3 Black str/et

Dia: caries

24.00.2017

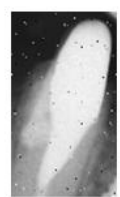

Figure 4. Watermarked images under rotation $\left(+10^{\circ}\right)$ and the extracted EPR and ROI watermarks: a host image with EPR1 and ROI1, b host image with EPR2 and ROI2

For scale attacks testing, the sizes of watermarked images were proportionally scaled with values $0.5,1.5,2.0$, and 3.0. Then for image restoration, a bicubic interpolation was applied. The obtained results are located in Table 2 .

The proposed algorithm provides the high robustness values of the ROI watermarks under the scale attacks. Thus, the error values are near $0.8-0.9 \%$ under the twofold increased scale that remains a visibility the best, especially in gray scale images (Figure 5). However, even a non-significant number of error bits in the EPR watermarks leads to visual degradation. The estimators for the twofold decreased scale are worse regarding to the twofold increased scale that is explained by the features of a bicubic interpolation. 


\begin{tabular}{|l|c|c|c|c|}
\hline \multicolumn{1}{|c|}{ Metric } & \multicolumn{4}{|c|}{ Proportional scaling } \\
& $0.5 \times$ & \multicolumn{1}{|c|}{$1.5 \times$} & $2.0 \times$ & $3.0 \times$ \\
BER, \% & 1.6 & 0.8 & 0.9 & 2.7 \\
& \multicolumn{5}{|c|}{ ROI1 } \\
SSIM, \% & 97.6 & 99.8 & 99.7 & 92.2 \\
PSNR, dB & 36.4 & 47.5 & 45.1 & 30.9 \\
BER, \% & 1.7 & 0.5 & 0.8 & 2.9 \\
& \multicolumn{7}{|c|}{ EPR2 } \\
BER, \% & 1.7 & 0.4 & 0.9 & 3.0 \\
& \multicolumn{4}{|c|}{ ROI2 } \\
SSIM, \% & 96.9 & 99.7 & 96.7 & 91.1 \\
PSNR, dB & 35.5 & 47.2 & 45.4 & 30.7 \\
BER, \% & 2.0 & 0.5 & 0.9 & 3.1 \\
\hline
\end{tabular}

Table 2. Estimations of the extracted EPR and ROI watermarks under scaling distortions

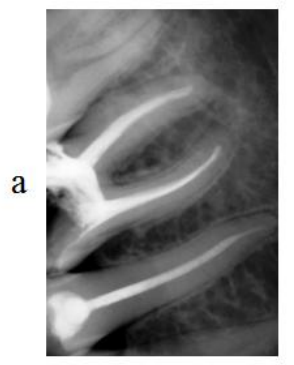

0015234

J.K.Johnson

m!le 52

12 WhiTe strget

Dia: periodontitis 24.02.17
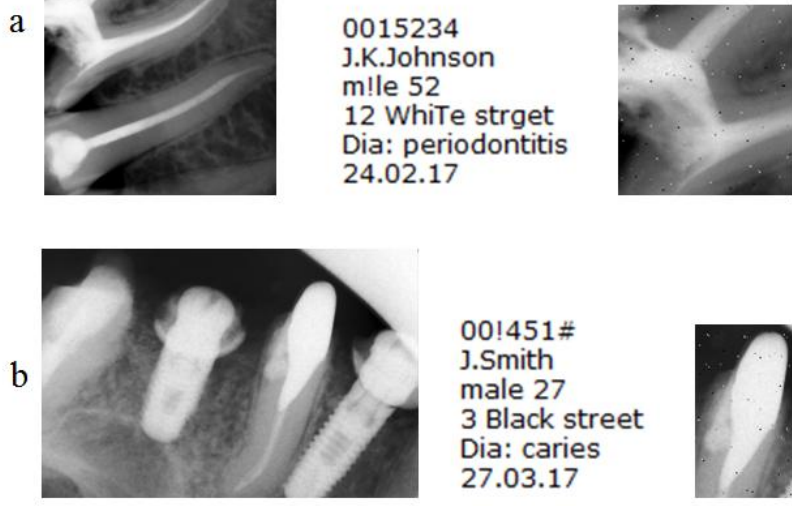

$00 ! 451$

J.Smith

male 27

3 Black street

Dia: caries

27.03.17

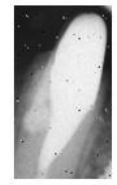

Figure 5. Watermarked images under proportional scaling $(2 \times)$ and the extracted EPR and ROI watermarks: a host image with EPR1 and ROI1, b host image with EPR2 and ROI2

The translation attacks were modeled by shifts of a host image in the vertical and/or horizontal directions on $5,8,12$, and 20 pixels. The estimators from Table 3 were obtained under the right and bottom shifts on a similar distance. A visual representation of this modeling is depicted in Figure 6.

\begin{tabular}{|l|c|c|c|c|}
\hline \multicolumn{1}{|c|}{ Metric } & \multicolumn{4}{|c|}{ Translation, pixel } \\
\hline & 5 & \multicolumn{4}{|c|}{8} & 12 & 20 \\
BER, \% & 4.5 & 6.6 & 9.0 & 9.8 \\
& \multicolumn{5}{|c|}{ ROI1 } \\
SSIM, \% & 89.0 & 84.7 & 80.5 & 78.1 \\
PSNR, dB & 31.1 & 28.4 & 26.0 & 21.5 \\
BER, \% & 4.1 & 7.0 & 8.4 & 11.3 \\
& \multicolumn{4}{|c|}{ EPR2 } \\
BER, \% & 4.1 & 6.7 & 8.2 & 12.5 \\
& \multicolumn{4}{|c|}{ ROI2 } \\
SSIM, \% & 90.3 & 84.8 & 81.1 & 78.2 \\
PSNR, dB & 31.3 & 27.9 & 25.6 & 22.0 \\
BER, \% & 4.4 & 6.8 & 9.2 & 11.9 \\
\hline
\end{tabular}

Table 3. Estimations of the extracted EPR and ROI watermarks under translation distortions

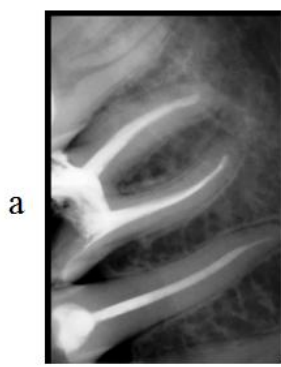

$001^{\perp} 234$

J.K.Johnson

maLe r2

12 White str\%et

Dia: periodonuitis

"4.02.17
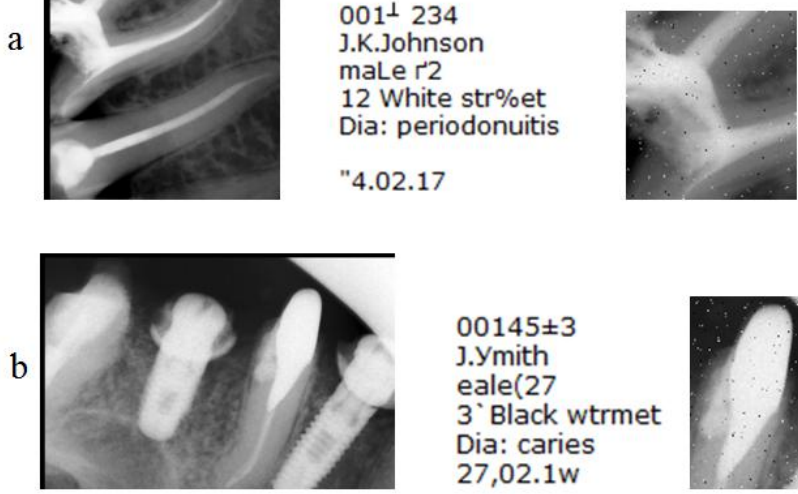

$00145 \pm 3$

J.Ymith

eale(27

3 'Black wtrmet

Dia: caries

27,02.1w

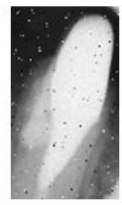

Figure 6. Watermarked images under translation on 12 pixels and the extracted EPR and ROI watermarks: a host image with

EPR1 and ROI1, b host image with EPR2 and ROI2

As it can be seen from the Table 3, the translation attacks distort an image stronger than the rotating and scaling attacks. Even for small values of shifts, a number of error bits exceeds $4 \%$. This is explained by a full loss of information because some pixels disappeared after shifts. (Note that a watermark is distributed by the DST uniformly in the RONI.)

Additionally to the affine transforms, the influence of JPEG compression was analyzed. The obtained results for the 5, 10, 20, and 30\% compression ratios are situated in Table 4 .

\begin{tabular}{|l|c|c|c|c|}
\hline \multicolumn{1}{|c|}{ Metric } & \multicolumn{4}{|c|}{ JPEG compression, \% } \\
\hline & 5 & 10 & 20 & 30 \\
BER, \% & 5.0 & $7.3 \quad \mid 11.2$ & 19.4 \\
& \multicolumn{5}{|c|}{ ROI1 } \\
SSIM, \% & 88.4 & 81.0 & 77.6 & 68.9 \\
PSNR, dB & 30.5 & 26.4 & 20.8 & 18.3 \\
BER, \% & 5.2 & 8.8 & 11.9 & 20.3 \\
& \multicolumn{4}{|c|}{ EPR2 } \\
BER, \% & 0.8 & $8.1 \quad 13.1$ & 20.6 \\
& \multicolumn{4}{|c|}{ ROI2 } \\
SSIM, \% & 89.1 & 80.6 & 77.1 & 69.9 \\
PSNR, dB & 30.7 & 25.8 & 22.1 & 17.8 \\
BER, \% & 4.8 & 8.9 & 12.2 & 19.5 \\
\hline
\end{tabular}

Table 4. Estimations of the extracted EPR and ROI watermarks under JPEG compression

The watermarked images under JPEG with compression ratio $20 \%$, as well as the extracted ROI-watermarks, are depicted in Figure 7. The experiments show that the proposed algorithm is sensitive to compression. However, the estimators remain suitable, viz. $12 \%$ of error losses in average under $20 \%$ of compression ratio. At the same time, the textual EPR watermarks suffer substantially and become unreadable under such compression degree. 


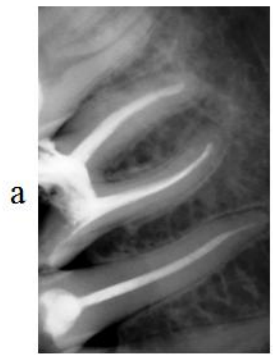

$0014 " 36$

J.K.Kohnsmj

$\mathrm{m}$ !hg 42

12 White streep

Dia:Operamdontitis

24@02.\#7
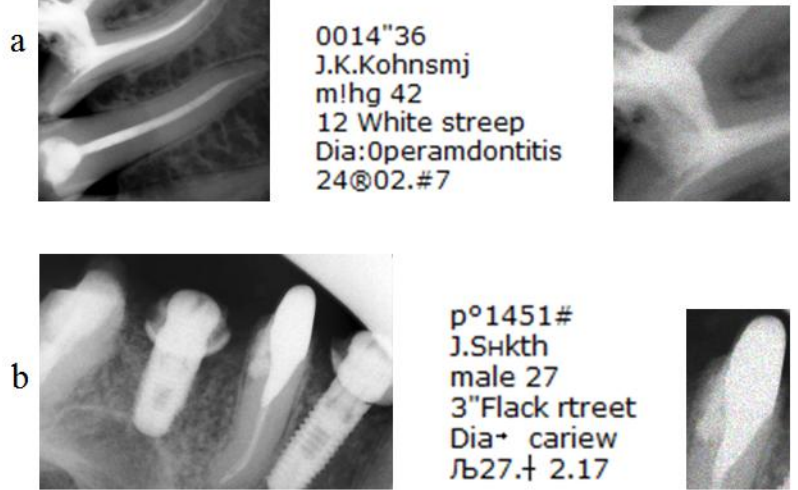

Figure 7. Watermarked images under JPEG with compression ratio $20 \%$ and the extracted EPR and ROI watermarks: a host image with EPR1 and ROI1, b host image with EPR2 and ROI2

Generally speaking, the obtained results are promising. The conventional post-processing of the watermarks and their host images may compensate the distortions caused by the widely distributed internet attacks.

\section{CONCLUSIONS}

In this research, a process of dental image watermarking was investigated. The application of the DST permitted to embed a huge data volume, involving the textual (the EPR) and image (the ROI) information in a host image with the restricted resolution. The proposed watermarking technique was tested on 40 dental gray scale images with various resolution. The experiments show the highest robustness to the rotations and proportional scaling (the BER mean values are 1.6-2.4\%) and the medium robustness to the translations and JPEG compression (the BER mean values are 7.9-12.4\%). The SSIM estimators are high and achieved $99.7 \%$ for the rotation and scaling distortions that shows good HVS properties.

\section{REFERENCES}

Acharya, R.U., Niranjan, U.C., Iyengar, S.S., Kannathal, N., Min, L.C., 2004. Simultaneous storage of patient information with medical images in the frequency domain. Computer Methods and Programs in Biomedicine, 76(1), pp. 13-19.

Badran, E.F., Sharkas, M.A., Attallah, O.A., 2009. Multiple watermark embedding scheme in wavelet-spatial domains based on ROI of medical images. In The National Radio Science Conference, Cairo, Egypt, pp. 1-8.

Blackledge, J., Al-Rawi, A., 2014. Stegacrypion of DICOM Metadata. In: The 25th Irish Signals \& Systems Conference and China-Ireland International Conference on Information and Communications Technologies, Limerick, Ireland, pp. 304-309.

Das, S., Kundu, M.K., 2012. Effective management of medical information through a novel blind watermarking technique. Journal of Medical Systems, 36(5), pp. 3339-3351.

DICOM. http://dicom.nema.org/ (30 Dec. 2016).
Dobbertin, H., Bosselaers, A., Preneel, B., 1996. RIPEMD-160: a strengthened version of RIPEMD. In: International Workshop on Fast Software Encryption, Cambridge, UK, pp. 71-82.

Easley, G., Labate, D., Lim, W.Q., 2008. Sparse directional image representations using the discrete shearlet transform. Applied and Computational Harmonic Analysis, 25(1), pp. 2546.

Easley, G.R., Labate, D., 2012. Image processing using shearlets, In: Kutyniok, G., Labate, D. (Eds.), Shearlets: Multiscale analysis for multivariate data, Springer, New York, Dordrecht, Heidelberg, London, pp. 283-325.

Fridrich, J., Goljan, M., Du, R., 2001. Invertible authentication. In: The SPIE, Security and Watermarking of Multimedia Contents III, Vol. 3971, San Jose, USA, pp. 197-208.

Giakoumaki, A., Pavlopoulos, S., Koutsouris, D., 2003. A medical image watermarking scheme based on wavelet transform. In: The 25th Annual International Conference of the IEEE Engineering in Medicine and Biology Society, Cancun, Mexico, pp. 856-859.

Guo, X., Zhuang, T.G., 2009. A region-based lossless watermarking scheme for enhancing security of medical data. Journal of Digital Imaging, 22(1), pp. 53-64.

Jianfeng, L., Wang, M., Junping, D., Huang, Q., Li, L., Chang, C.C., 2015. Multiple watermark scheme based on DWT-DCT quantization for medical images. Journal of Information Hiding and Multimedia Signal Processing, 6(3), pp. 458-72.

Kong, X., Feng, R., 2001. Watermarking medical signals for telemedicine. IEEE Transactions on. Information Technology in Biomedicine, 5(3), pp. 195-201.

Kumar, S., 2015. A comparative study of transform based on secure image steganography. International Journal of Computer and Communication Engineering, 4(2), pp. 107-116.

Makbol, N.M., Khoo, B.E., 2014. A new robust and secure digital image watermarking scheme based on the integer wavelet transform and singular value decomposition. Digital Signal Processing, 33, pp. 134-147.

Manasrah, T., Al-Haj, A., 2008. Management of medical images using wavelets-based multi-watermarking algorithm. In: The International Conference on Innovations in Information Technology, Al-Ain, UAE, pp. 697-701.

Mohananthini, N., Yamuna, G., 2015. A study of DWT-SVD based multiple watermarking scheme for medical images. International Journal of Network Security, 17(5), pp. 558-568.

Nambakhsh, M.S., Ahmadian, A., Zaidi, H., 2011. A contextual based double watermarking of PET images by patient ID and ECG signal. Computer Methods and Programs in Biomedicine, 104(3), pp. 418-25.

Nyeem, H., Boles, W., Boyd, C., 2013. A review of medical image watermarking requirements for teleradiology. Journal of Digital Imaging, 26(2), pp. 326-343.

Nyeem, H., Boles, W., Boyd, C., 2015. Content-independent embedding scheme for multi-modal medical image watermarking. BioMedical Engineering, OnLine 14:7, DOI: $10.1186 / 1475-925 X-14-7$ 
Osborne, D., Abbott, D., Sorell, M., Rogers, D., 2004. Multiple embedding using robust watermarks for wireless medical images. In: The 3rd International Conference on Mobile and ubiquitous multimedia, College Park, Maryland, USA, pp. 245250 .

Pizer, S.M., Amburn, E.P., Austin, J.D., Cromartie, R., Geselowitz, A., Greer, T., ter Haar Romeny, B., Zimmerman, J.B., Zuiderveld, K., 1987. Adaptive histogram equalization and its variations. Computer vision, graphics, and image processing, 39(3), pp. 355-368.

Puech, W., Rodrigues, J.M., 2004. A new crypto-watermarking method for medical images safe transfer. In: The $12^{\text {th }}$ European Signal Processing Conference, Vienna, Austria, pp. 1481-1484.

Wakatani, A., 2002. Digital watermarking for ROI medical images by using compressed signature image. In: The 35th Annual Hawaii International Conference on System Sciences, Big Island, Hawaii, Vol. 6, pp. 157-163.

Wang, X.Y., Liu, Y.N., Xu, H., Wang, A.L., Yang, H.Y., 2016. Blind optimum detector for robust image watermarking in nonsubsampled shearlet domain. Information Sciences, 372, pp. 634-654.

Yi, S., Labate, D., Easley, G.R., Krim, H., 2009. A Shearlet approach to edge analysis and detection. IEEE Transaction on Image Processing, 18(5), pp. 929-941. 